\title{
ON THE ACCURACY OF DOUBLE SCATTERING APPROXIMATION FOR ATMOSPHERIC POLARIZATION COMPUTATIONS
}

\author{
Sergey V. Korkin ${ }^{1,2}$, Alexei I. Lyapustin ${ }^{3}$, Alexander L. Marshak ${ }^{3}$ \\ ${ }^{1}$ Universities Space Research Association, 10211, Wincopin Circle, Columbia, MD, 21044, USA; \\ ${ }^{2}$ NASA Goddard Space Flight Center, Greenbelt, code 614.4, MD, 20771, USA; \\ ${ }^{3}$ NASA Goddard Space Flight Center, Greenbelt, code 613.2, MD, 20771, USA.
}

\begin{abstract}
Interpretation of multi-angle spectro-polarimetric data in remote sensing of atmospheric aerosols require fast and accurate methods of solving the vector radiative transfer equation (VRTE). The single and double scattering approximations could provide an analytical framework for the inversion algorithms and are relatively fast, however accuracy assessments of these approximations for the aerosol atmospheres in the atmospheric window channels have been missing. This paper provides such analysis for a vertically homogeneous aerosol atmosphere with weak and strong asymmetry of scattering. In both cases, the double scattering approximation gives a high accuracy result (relative error $\sim 0.2 \%$ ) only for the low optical path $\sim 10^{-2}$. As the error rapidly grows with optical thickness, a full VRTE solution is required for the practical remote sensing analysis. It is shown that the scattering anisotropy is not important at low optical thicknesses neither for reflected nor for transmitted polarization components of radiation.
\end{abstract}

Keywords: vector radiative transfer; secondary scattering; arbitrary scattering law.

\section{Introduction}

A complete solution of the vector radiative transfer equation (VRTE) is time-consuming. At the same time, interpretation of the airborne (Chowdhary et al., 2001) as well as of the current (Dubovik et al., 2011) and future satellite multi-angle spectro-polarimetric measurements requires fast and sufficiently accurate methods of VRTE solution. The polarimetric accuracy of the POLDER-type instrument is $\sim 2 \%$ (http://smsc.cnes.fr /POLDER/A_calibration.htm). The more precise systems target the accuracy of $\sim 0.2 \%$ for "the measured orthogonal polarization states" (Mishchenko et al., 2007) or the degree of linear polarization (Diner et al. 2007, 2010).

A fast approximate VRTE solution can be obtained using the first two orders of scattering. These approximations offer convenience by providing an analytical framework for the inversion algorithms. The original derivation for the first two scattering orders for Rayleigh scattering was given by Hammad and Chapman (1939), Hammad (1948) with numerical results presented in (Hammad, 1953). Later, Dave (1964) gave a solution and numerical analysis for the first three orders of Rayleigh scattering. In his 
study, Dave (1964, p.313) concluded that “... it is sufficient to consider only primary and secondary scattering when the scattering optical thickness is 0.05. But the number of necessary orders of scattering increases very rapidly with optical thickness and is about three and eight for optical thickness 0.1 and 1.0 , respectively" or higher to achieve $0.1 \%$ level of accuracy.

Using the adding-doubling method, Hovenier (1971) derived equations for the first few orders of scattering for the plane-parallel homogeneous atmosphere. This work used an analytical integration over the optical thickness and numerical integration over the angle. From analysis for the Rayleigh and two types of Henyey-Greenstein phase matrices and optical thickness in the range 0.25 to 64 , it was concluded that "approximations, based on polarization by single scattering only ... lead to large errors. Adding polarization by second order scattering gives only a small improvement" for the intensity and degree of linear polarization (Hovenier, 1971).

Kawabata and Ueno (1988) developed algorithm for the vertically inhomogeneous media to compute the scalar intensity using the first three orders of scattering. This algorithm was advanced by Natraj and Spurr (2007) who provided the vector solution for the first two orders of scattering using the generalized spherical functions formalism (Gelfand et al., 1963; Siewert, 2000). Natraj and Spurr (2007) gave a detailed accuracy study of the approximate solution in application to the oxygen A-band, reporting that "results are exact in the center of strong lines and most inaccurate ( $30 \%$ error in the Stokes parameter $Q$ ) in the continuum".

Although the theoretical derivations of the low orders of scattering VRTE approximation were previously reported, their accuracy evaluation for the aerosol atmospheres in the atmospheric window channels has been missing. This paper presents an accuracy study for an arbitrary anisotropic scattering matrix and both reflected and transmitted radiation. We pursue a practical target of $0.2-0.5 \%$ in computation of polarized components of intensity. This level of accuracy dramatically limits the possible range of optical depths to Dave's (1964) assessment of 0.05. For this reason, this paper only considers the homogeneous case although the computational method could be generalized to include atmospheric vertical structure. For the same reason, the main accuracy analysis focuses on a conservative scattering case which provides the strongest constraint on the range of optical depths given the target accuracy.

This paper is structured as follows: the statement of the problem, the algorithm of solution and computational relations, used in the paper, are given in Section (2). The definition of the cases, numerical results and discussion are presented in Section (3). The paper is concluded with a brief summary.

\section{Computational Relations}

We are considering the VRTE boundary problem without thermal sources for the homogeneous plane-parallel atmosphere over a black surface (Chandrasekhar, 1950). The VRTE solution is 
decomposed into azimuthal Fourier series following Siewert (2000). The single scattering (SS) solution for the $m$-th harmonic can be obtained following van de Hulst (1948):

$$
\overrightarrow{\mathrm{L}}_{1}^{m}(\tau, \mu)= \begin{cases}\frac{\omega_{0}}{4 \pi} \frac{\mu_{0}}{\mu_{0}-\mu}\left(\exp \left(-\frac{\tau}{\mu_{0}}\right)-\exp \left(-\frac{\tau}{\mu}\right)\right) \overrightarrow{\mathrm{P}}^{m}\left(\mu, \mu_{0}\right) \overrightarrow{\mathrm{L}}_{0}, & \mu>0 ; \\ \frac{\omega_{0}}{4 \pi} \frac{\tau}{\mu} \exp \left(-\frac{\tau}{\mu}\right) \overrightarrow{\mathrm{P}}^{m}\left(\mu, \mu_{0}\right) \overrightarrow{\mathrm{L}}_{0}, & \mu \rightarrow \mu_{0} ; \\ \frac{\omega_{0}}{4 \pi} \frac{\mu_{0}}{\mu_{0}-\mu}\left(\exp \left(-\frac{\tau}{\mu_{0}}\right)-\exp \left(\frac{\tau_{0}-\tau}{\mu}-\frac{\tau_{0}}{\mu_{0}}\right)\right) \overrightarrow{\mathrm{P}}^{m}\left(\mu, \mu_{0}\right) \overrightarrow{\mathrm{L}}_{0}, & \mu<0 .\end{cases}
$$

The following notations are used above: $\overrightarrow{\mathrm{L}}=\left[\begin{array}{llll}I & Q & U & V\end{array}\right]$ is the Stokes vector, $\overrightarrow{\mathrm{L}}_{0}$ is the initial vector for solar irradiance, the overhead symbol " $\leftrightarrow$ " indicates square matrices, $\tau\left(0 \leq \tau \leq \tau_{0}\right)$ is the optical thickness, and $\omega_{0}$ is the single scattering albedo (SSA). The directions are given by the solar (SZA, $\left.\theta_{0}\right)$ and view $($ VZA, $\theta)$ zenith angles $(\mu=\cos \theta)$. The positive $\left(\mu_{+}=\mu>0\right)$ and negative directions correspond to transmitted and reflected light, respectively. The $m$-th Fourier term of the phase matrix is

$$
\overrightarrow{\mathrm{P}}^{m}\left(\mu, \mu^{\prime}\right)=\sum_{k=m}^{K}(2 k+1) \ddot{\Pi}_{k}^{m}(\mu) \vec{p}_{k} \ddot{\Pi}_{k}^{m}\left(\mu^{\prime}\right)
$$

where $\vec{p}_{k}$ is defined by the greek-constants (de Rooij and van der Stap, 1984). $\ddot{\Pi}_{k}^{m}(\mu)$ contains $P_{k}^{m}(\mu)$, $R_{k}^{m}(\mu)$ and $T_{k}^{m}(\mu)$ polynomials related to generalized spherical functions (Gelfand et al., 1963; Siewert, 2000). $K$ is the maximal considered order of the polynomials. The azimuth-dependent solution is obtained from Eq.(1) after summation Fourier series over $m$.

Using Eq.(1) as a source function in VRTE and applying analytical integration over $\tau$ (Hovenier, 1971), the following result for the second order scattering $\overrightarrow{\mathrm{L}}_{2}^{m}(\tau, \mu)$ can be obtained:

- Reflected radiance:

$$
\begin{gathered}
\overrightarrow{\mathrm{L}}_{2}^{m}(\tau, \mu<0)=\frac{\omega_{0}^{2} \mu_{0}}{8 \pi}\left(\int _ { 0 } ^ { 1 } \frac { \ddot { \mathrm { P } } ^ { m } ( \mu , \mu _ { + } ^ { \prime } ) \vec { \mathrm { P } } ^ { m } ( \mu _ { + } ^ { \prime } , \mu _ { 0 } ) \vec { \mathrm { L } } _ { 0 } } { \mu _ { 0 } - \mu _ { + } ^ { \prime } } \left[\frac{\mu_{0}}{\mu_{0}-\mu} \exp \left(-\frac{\tau}{\mu_{0}}\right)\left\{1-\exp \left(\frac{\mu_{0}-\mu}{\mu_{0} \mu} \Delta \tau\right)\right\}-\right.\right. \\
\left.-\frac{\mu_{+}^{\prime}}{\mu_{+}^{\prime}-\mu} \exp \left(-\frac{\tau}{\mu_{+}^{\prime}}\right)\left\{1-\exp \left(\frac{\mu_{+}^{\prime}-\mu}{\mu_{+}^{\prime} \mu} \Delta \tau\right)\right\}\right] d \mu_{+}^{\prime}+\int_{-1}^{0} \frac{\overrightarrow{\mathrm{P}}^{m}\left(\mu, \mu_{-}^{\prime}\right) \overrightarrow{\mathrm{P}}^{m}\left(\mu_{-}^{\prime}, \mu_{0}\right) \overrightarrow{\mathrm{L}}_{0}}{\mu_{0}-\mu_{-}^{\prime}}\left[\frac{\mu_{0}}{\mu_{0}-\mu} \exp \left(-\frac{\tau}{\mu_{0}}\right) \times\right. \\
\left.\left.\times\left\{1-\exp \left(\frac{\mu_{0}-\mu}{\mu \mu_{0}} \Delta \tau\right)\right\}-\exp \left(-\frac{\tau_{0}}{\mu_{0}}\right) \frac{\mu_{-}^{\prime}}{\mu_{-}^{\prime}-\mu}\left\{\exp \left(\frac{\Delta \tau}{\mu_{-}^{\prime}}\right)-\exp \left(\frac{\Delta \tau}{\mu}\right)\right\}\right] d \mu_{-}^{\prime}\right) .
\end{gathered}
$$

- Transmitted radiance:

$$
\overrightarrow{\mathrm{L}}_{2}^{r}(\tau, \mu>0)=\frac{\omega_{0}^{2} \mu_{0}}{8 \pi}\left(\int _ { 0 } ^ { 1 } \frac { \ddot { \mathrm { P } } ^ { m } ( \mu , \mu _ { + } ^ { \prime } ) \vec { \mathrm { P } } ^ { m } ( \mu _ { + } ^ { \prime } , \mu _ { 0 } ) \vec { \mathrm { L } } _ { 0 } } { \mu _ { 0 } - \mu _ { + } ^ { \prime } } \left[\frac{\mu_{0}}{\mu_{0}-\mu}\left\{\exp \left(-\frac{\tau}{\mu_{0}}\right)-\exp \left(-\frac{\tau}{\mu}\right)\right\}-\right.\right.
$$




$$
\begin{aligned}
\left.-\frac{\mu_{+}^{\prime}}{\mu_{+}^{\prime}-\mu}\left\{\exp \left(-\frac{\tau}{\mu_{+}^{\prime}}\right)-\exp \left(-\frac{\tau}{\mu}\right)\right\}\right] d \mu_{+}^{\prime}+\int_{-1}^{0} \frac{\overrightarrow{\mathrm{P}}^{m}\left(\mu, \mu_{-}^{\prime}\right) \overrightarrow{\mathrm{P}}^{m}\left(\mu_{-}^{\prime} \mu_{0}\right) \overrightarrow{\mathrm{L}}_{0}}{\mu_{0}-\mu_{-}^{\prime}}\left[\frac{\mu_{0}}{\mu_{0}-\mu}\left\{\exp \left(-\frac{\tau}{\mu_{0}}\right)-\exp \left(-\frac{\tau}{\mu}\right)\right\}-\right. \\
\left.\left.-\exp \left(-\frac{\tau_{0}}{\mu_{0}}\right) \frac{\mu_{-}^{\prime}}{\mu_{-}^{\prime}-\mu}\left\{\exp \left(\frac{\Delta \tau}{\mu_{-}^{\prime}}\right)-\exp \left(\frac{\tau_{0}}{\mu_{-}^{\prime}}\right) \exp \left(-\frac{\tau}{\mu}\right)\right\}\right] d \mu_{-}^{\prime}\right), \text { where } \Delta \tau=\tau_{0}-\tau .
\end{aligned}
$$

In the developed code, a gaussian quadrature is used to perform integration in Eqs. (3) and (4). The singular points $1 /\left(\mu^{\prime}-\mu\right)$ for $\mu \rightarrow \mu^{\prime}$ can be handled either by changing the order of quadrature to avoid view directions or by expanding the exponents in Taylor series. Below, the term "double scattered" (DS) will be used to describe contribution from the first two orders of scattering, $\overrightarrow{\mathrm{L}}_{1}^{m}(\tau, \mu)+\overrightarrow{\mathrm{L}}_{2}^{m}(\tau, \mu)$ (Eqs. (1), (3), (4)).

\section{Numerical Results and Discussion}

The accuracy of SS and DS solutions was investigated using three types of scattering matrices with different scattering anisotropy. The first one is Rayleigh scattering matrix (Coulson et al., 1960) which has only two non zero expansion moments. The second one represents the fine spherical nonabsorbing aerosol particles described by the gamma distribution with effective radius $0.2 \mu \mathrm{m}$, variance $0.07 \mu \mathrm{m}$, and real refractive index 1.44 (de Rooij and van der Stap, 1984). The scattering matrix at the wavelength $0.951 \mu \mathrm{m}$ has the average scattering cosine ASC $=\left[\vec{p}_{k=1}\right]_{1,1}=0.485$ (see Eq.(2)) and $K=11$ expansion terms. It was used by Evans and Stephens (1991) and is provided at http://nit.colorado.edu/. The third one, used in the code comparison study of Kokhanovsky et al. (2010) and available at www.iup.physik.uni-bremen.de/ alexk, represents the coarse aerosol fraction described by a lognormal size distribution with effective radius $0.3 \mu \mathrm{m}$, variance $0.92 \mu \mathrm{m}$, and real refractive index 1.385 . It is characterized by the ASC $=0.793$ and $K=918$ expansion moments. All $K$ moments were kept in our computations. The phase matrices for aerosol cases are shown in Figure 1.

The accuracy of the result can be estimated using the relative error, e.g. $E_{l}=(I-\tilde{I}) 100 / I[\%]$, where $I$ and $\tilde{I}$ are the accurate and the approximate solutions, respectively. Because the polarization components $Q, U, V$ may take zero values at certain points whose location is a priori unknown, we will further use the Chandrasekhar's vector with the first two components defined as $I_{l}=(I+Q) / 2, I_{r}=(I-$ $Q) / 2 . I_{l}$ and $I_{r}$ represent the parallel and perpendicular components of the polarized light intensity (Chandrasekhar, 1950; van de Hulst, 1957) which are non-negative. $I_{l}$ may take a zero value only in the case of $100 \%$ linear polarization that rarely happens in the atmosphere. Thus, the accuracy is evaluated below using the following relative error [\%]:

$$
E_{l, r}=\left(I_{l, r}-\tilde{I}_{l, r}\right) 100 / I_{l, r} .
$$


The accuracy of SS and DS solutions in this work was established by comparison with the vector code MVDOM (Budak et al., 2010). The relative errors $E_{l}$ and $E_{r}$ for the case of conservative Rayleigh scattering for the reflected and transmitted radiance were studied as a function of VZA for two solar zenith angles $\mathrm{SZA}=20^{\circ}, 60^{\circ}$ and five relative azimuths $\varphi=0^{\circ}, 45^{\circ}, 90^{\circ}, 135^{\circ}, 180^{\circ}$. Two optical depths $\tau$ $=0.01$ and $\tau=0.05$ were chosen as in Dave (1964). The results obtained are in good agreement with those from the previous studies (Dave, 1964). At low optical depth, the error of SS approximation can be as high as several percent. It reaches $100 \%$ at $90^{\circ}$ to the light incidence direction for the parallel component of intensity. In this direction, the amplitude of scattering of $I_{l}$ component is zero in the first order of scattering, and thus all of $I_{l}$ signal is created by the multiple scattering. This can be shown formally using the scattering matrix in Chandrasekhar's basis, which is given by the following expression (Chandrasekhar, 1950, Chapter 1, Eq.(201))

$$
\ddot{p}_{C h}(\gamma)=1.5 \operatorname{diag}\left[\gamma^{2} 1 / \gamma \gamma\right]
$$

At $90^{\circ}$ scattering angle, its cosine is zero $(\gamma=0)$ which cancels the first order scattering contribution for the $I_{l}$ component. With addition of the second scattering order, the error drops below 1-1.5\% except when close to the $90^{\circ}$ scattering angle when the maximal error reaches $12-18 \%$ at $\tau=0.01$ and 0.05 , respectively.

The error analysis for the fine aerosol fraction is presented in Figure 2 for the same view geometry. The top and bottom plots show the reflected and transmitted radiation, respectively. The dash and solid lines correspond to $\mathrm{SZA}=20^{\circ}$ and $60^{\circ}$, respectively, and different azimuths are indicated by different colors. Compared to the Rayleigh case, the errors are a factor of $\sim 2$ higher at the same optical thickness. The average errors $E_{r}$ and $E_{l}$ of the DS solution are approximately $0.6-0.8 \%$ at $\tau=0.01$ and 1.8 $2.5 \%$ at $\tau=0.05$, while the errors of the SS solution are about a factor of 8 higher. The maximal error for the parallel component of light is shifted from $90^{\circ}$ towards larger scattering angles, which follows from the Mie theory (van de Hulst, 1957, p. 147, figure 23).

Figure 3 repeats the error analysis for the coarse aerosol model. In this case, $N=600$ ordinates were used in computations with $K=918$ expansion terms. The error decreases as compared to both the fine aerosol model (Figure 2) and Rayleigh case. It still remains at the level of several percent in the SS approximation, but drops down to $0.2-0.3 \%$ and $1-1.5 \%$ in the DS approximation at $\tau=0.01$ and 0.05 , respectively.

The above analysis was conducted for the conservative scattering case. An aerosol absorption reduces the number of scattering events thereby increasing the accuracy of SS and DS approximations. However, the error changes insignificantly within the realistic range of aerosol SSA values $0.8-1$.

The analyzed approximate solution does not describe well the neutral points of polarization of the clear sky (Chandrasekhar, 1950). These points appear only in the DS approximation, but are shifted with respect to the true position (Hovenier, 1971). For example, at $\tau=0.2$, the Babinet and Brewster points ( $\varphi=$ 
$0^{\circ}$ ) are shifted towards incidence direction by approximately $3^{\circ}$ and $5^{\circ}$, respectively, while the Arago point $\left(\mathrm{SZA}=78.5^{\circ}\right.$ and $\varphi=180^{\circ}$ ) is shifted $5^{\circ}$ towards the horizon.

The results presented above allow us to extend conclusion of Dave, made for the Rayleigh case, to the aerosol atmospheres. Namely, the first two scattering orders provide the high accuracy of polarization computations $\sim 0.2-0.5 \%$ only when the media is optically thin $(\tau<0.01)$ or there is a significant gaseous absorption which essentially eliminates higher orders of scattering (Natraj and Spurr, 2007). In the typical case of aerosol remote sensing in the atmospheric window channels, the accuracy of the DS solution deteriorates rapidly with the optical thickness making it impractical for the data analysis.

One interesting finding of this analysis is a weak dependence of the relative errors $E_{l}$ and $E_{r}$, only within a factor of $2-4$, among the three considered cases with the large difference in the scattering matrix anisotropy. One explanation is that all considered examples were generated for the optically thin media $(\tau \leq 0.1)$ where the first two scattering orders dominate the signal regardless of the scattering anisotropy. To confirm this statement, a numerical experiment was performed with the Monte-Carlo code (Marshak and Davis, p. 264) simulating the transfer of photons along a single line (1D transport). The photon can scatter forward with the probability $p_{+}=0.5 \cdot(1+g)$ or backward with the probability $p_{-}=0.5 \cdot(1-g)$, where $g$ is the asymmetry parameter, or ASC. Figure 4 shows the number of photons scattered once $\left(n_{1}\right.$, blue line) and twice ( $n_{2}$, green line) and their sum $\left(n_{1}+n_{2}\right.$, red line) as a fraction of the total number of scattering events $(N)$. These fractions correspond to the reflected (transmitted) radiation calculated using the first order or the two orders of scattering. The superscripts " $r$ " and " $t$ " denote the reflected and transmitted radiation, which was computed for $\mathrm{SSA}=1$ and three values of optical depth $\tau=0.1,1,10$. The ratio between the forward and backward scattering $(1+g) /(1-g)$ ranges from 5.67 to $9,19,199$ for $g=0.7$, $0.8,0.9,0.99$ respectively. The left plots of Figure 4 at $\tau=0.1$ shows that the relative contribution of the first two scattering orders $\left(n_{l} / N\right.$ and $\left.n_{2} / N\right)$ changes little while the asymmetry of phase function changes significantly with $g$. This, however, is not the case when higher orders of scattering become prominent at larger optical depths $\tau=1,10$. Note that for larger optical depths (right panels), the single scattering contribution to the total reflected or transmitted radiation decreases with the increase of the phase function anisotropy.

All of our previous results considered an optically thin case. At the opposite end of very high optical depths, the accuracy of SS and DS solutions for $Q$ and $U$ components rises again because the high scattering orders with alternating sign from rotation of the reference plane effectively cancel each other. This effect is illustrated in Figure 5 showing Stokes components for radiation reflected from a cloud with $\tau=50$ (SSA=1). The "cloud" model (Kokhanovsky et al., 2010), available at www.iup.physik.unibremen.de/ alexk, is characterized by an effective radius $5 \mu \mathrm{m}$, variance $0.4 \mu \mathrm{m}$, and maximal droplet size of $100 \mu \mathrm{m}$. Although the approximate result has a rather low accuracy, the DS-solution captures a large part of $Q$ and $U$ components, unlike $I$ and $V$ which require accumulation of all orders of scattering. 


\section{Conclusion}

This paper studied the accuracy for the first two scattering orders using code MVDOM as a reference. Although the previous studies conducted for Rayleigh scattering (Dave, 1963) showed that the DS approximation has a good accuracy only at low optical depths $(\tau<0.05)$, it was not clear whether this conclusion holds for the aerosol atmospheres with higher scattering anisotropy which tends to reduce the effect of light polarization on the total intensity (Mishchenko et al., 2006). Our analysis, which included two aerosol scattering matrices with weak and strong anisotropy, corroborated Dave's conclusion showing that DS approximation provides an accuracy of better than $0.2-0.5 \%$ at $\tau \sim 0.01$, and $1-2 \%$ at $\tau \sim 0.05$, however a full multiple scattering solution is required at higher optical depths. At low optical depths, this error has a weak dependence on the anisotropy of the scattering matrix.

Although we demonstrated that the DS approximation cannot be used at $\tau>0.05$, it has a certain didactic value as an approximate solution for the degree of polarization at the near $90^{\circ}$ scattering angles as well as for the $V$-component of the Stokes vector. In these cases, the primary scattering gives a zero contribution (e.g., see Figure 5), and the signal is created by the higher orders of scattering. Presently, both theory and passive remote sensing applications pay little attention to the atmospheric ellipticity as $V$ signal is much weaker than the other components of the Stokes vector. On the other hand, as well as the total intensity, $V$-component does not depend on the choice of the reference plane, and its information content is not reduced by the higher orders of scattering contrary to the $Q$ and $U$ components. The DS approximation thus may serve as a useful constraint for the $V$-component in accurate computations.

\section{References}

1. Budak V.P., Klyuykov D.A., Korkin S.V., 2010: Convergence acceleration of radiative transfer equation solution at strongly anisotropic scattering. In: Light Scattering Reviews 5. Single Light Scattering and Radiative Transfer. Ed. A.A. Kokhanovsky. Chichester: Springer, pp.147 - 204.

2. Chandrasekhar S., 1950: Radiative transfer. London: Oxford University Press.

3. Chowdhary J., Cairns B., Mishenko M., and Travis L., 2001: Retrieval of aerosol properties over ocean using multispectral and multiangle photopolarimetric measurments from the Research Scanning Polarimeter. Geophys. Res. Lett., 28, pp. 383 - 397

4. Coulson K.L., Dave J.V., and Sekera Z., 1960: Tables Related to Radiation Emerging from a Planetary Atmosphere with Rayleigh Scattering. Berkeley: University of California Press.

5. Dave J.V., 1964: Importance of higher order scattering in a molecular atmosphere. JOSA, 54, 3, pp. $307-315$. 
6. Diner D. J., Davis A., Hancock B., Gutt G., Chipman R. A., and Cairns B., 2007: Dual photoelastic modulator-based polarimetric imaging concept for aerosol remote sensing. Applied Optics, 46, 35, pp. $8428-8445$

7. Diner D. J., Davis A., Hancock B., Geier S., Rheingans B., Jovanovic V., Bull M., Rider D. M., Chipman R. A., Mahler A.-B., and McClain S. C., 2010: First results from a dual photoelastic modulator-based polarimetric camera. Applied Optics, 49, pp. 2929-2946.

8. Dubovik, O., M. Herman, A. Holdak et al,, 2011: Statistically optimized inversion algorithm for enhanced retrieval of aerosol properties from spectral multi-angle polarimetric satellite observations, Atmos. Meas. Tech., 4, pp. 975-1018.

9. Evans K.F., Stephens G.L., 1991: A new polarized atmospheric radiative transfer model, JQSRT, 46, 5, pp. $413-423$.

10. Gelfand, I.M., R.A. Minlos, Z.Ya. Shapiro, 1963: Representations of the Rotation and Lorentz Groups and their Applications, Oxford: Pergamon Press.

11. Hammad A., Chapman S., 1939: The primary and secondary scattering of sunlight in a planestratified atmosphere of uniform composition. Phil. Mag., 28, 186, pp.99-110.

12. Hammad A., 1948: The primary and secondary scattering of a sunlight in a plane-stratified atmosphere of uniform composition. Astoph. J., 108, pp.338 - 346.

13. Hammad A., 1953: Calculation of the polarization of a sunlit sky composed of pure air molecules. JOSA, 43, 3, pp. $184-187$.

14. Hovenier J.W., 1971: Multiple scattering of polarized light in planetary atmospheres. Astron. Astroph., 13, pp.7-29.

15. van de Hulst H.C., 1948: Scattering in planetary atmosphere. Astroph. J., 107, pp.220 - 246.

16. van de Hulst H.C., 1957: Light Scattering by Small Particles. New-York: Wiley.

17. Kawabata K., Ueno S., 1988: The first three orders of scattering in vertically inhomogeneous scattering-absorbing media. Astrophys. Space Sc., 150, pp.327 - 344.

18. Kokhanovsky A.A., Budak V.P., Cornet C., Duan M., Emde C., Katsev I.L., Klyukov D.A., Korkin S.V., C-Labonnote L., Mayer B., Min Q., Nakajima T., Ota Y., Prikhach A.S., Rozanov V.V., Yokota T., Zege E.P., 2010: Benchmark results in vector atmospheric radiative transfer. 
JQSRT, 111, pp.1931-1946.

19. Marshak A., Davis A.B. (Eds.), 2005: 3D Radiative Transfer in Cloudy Atmospheres. Berlin: Springer-Verlag.

20. Mishchenko M.I., Travis L.D., Lacis A.A., 2006: Multiple scattering of light by particles: radiative transfer and coherent backscattering. Cambridge: Cambridge University Press.

21. Mishchenko M.I., Cairns B., Kopp G., Schueler C.F., Fafaul B.A., Hansen J.E., Hooker R.J., Itchkawich T., Maring H.B., and Travis L.D., 2007: Accurate monitoring of terrestrial aerosols and total solar irradiance. BAMS, 88, 5, pp.677-691.

22. Natraj V., Spurr R.J.D., 2007: A fast pseudo-spherical two orders of scattering model to account for polarization in vertically inhomogeneous scattering-absorbing media. JQSRT, 107, pp.263 - 293.

23. de Rooij W. A., van der Stap C. C. A. H., 1984: Expansion of Mie Scattering Matrices in Generalized Spherical Functions. Astronomy and Astrophysics, 131, pp.237-248.

24. Siewert C.E., 2000: A discrete-ordinates solution for radiative-transfer models that include polarization effects. JQSRT, 64, pp.227-254. 
Figure 1. Phase matrices for aerosol cases defined in the paper. The maximal values of the phase functions $a_{l}$ of the fine and coarse fractions are approximately 4 and 1500 respectively; $a_{1}, a_{3}$, $b_{1}, b_{2}$ are the $[1,1],[3,3],[1,2]$ and $[3,4]$ elements of the phase matrix respectively.

Figure 2. Relative error (\%) for the parallel (Figures 2(a) and 2(b)) and perpendicular (Figures 2(c) and 2(d)) components of the reflected $\left(\mathrm{VZA}=90^{\circ} \ldots 180^{\circ}\right)$ and transmitted $\left(\mathrm{VZA}=0^{\circ} \ldots 90^{\circ}\right)$ radiation for the fine aerosol model. The error is computed for five different azimuthal angles $\varphi=0^{\circ}, 45^{\circ}, 90^{\circ}, 135^{\circ}, 180^{\circ}$ shown by different colors (refer to the legend, Figure 2(e)), and two solar zenith angles $\mathrm{SZA}=20^{\circ}$ (dash lines) and $60^{\circ}$ (solid lines). The results are shown for two values of optical thickness, $\tau=0.01$ and $\tau=0.05$ at SSA $=1$. Figures 2(a) and 2(c) show the result for the single scattering approximation. Figures 2(b) and 2(d) show the result for the double scattering approximation.

Figure 3. The same as in Figure 2 but for the coarse aerosol fraction. The legend is shown in Fugure 2(e).

Figure 4. The Monte-Carlo simulations of the number of single scattered photons ( $n_{1}$, blue line), re-scattered photons $\left(n_{2}\right.$, green line $)$ and their sum $\left(n_{1}+n_{2}\right.$, red line) as a fraction of all scattered photons $(N)$. SSA $=1$ (see sec. 3). The superscripts " $r$ " and " $t$ " refer to the reflected and transmitted photons respectively. The ASC is the average scattering cosine.

Figure 5. I, $Q, U$, and $V$ components of the Stokes vector of reflected light for a cloud (Kokhanovsky et al., 2010) with $\tau=50$ (see sec. 3 ). The view geometry is described by $\mathrm{SZA}=30^{\circ}, \varphi=45^{\circ}$ (Fig. 5a), and $\varphi=135^{\circ}$ (Fig. 5b). The green, blue and red lines represent the single scattering (SS), double scattering (DS), and the full multiple scattering (MS) solutions. 

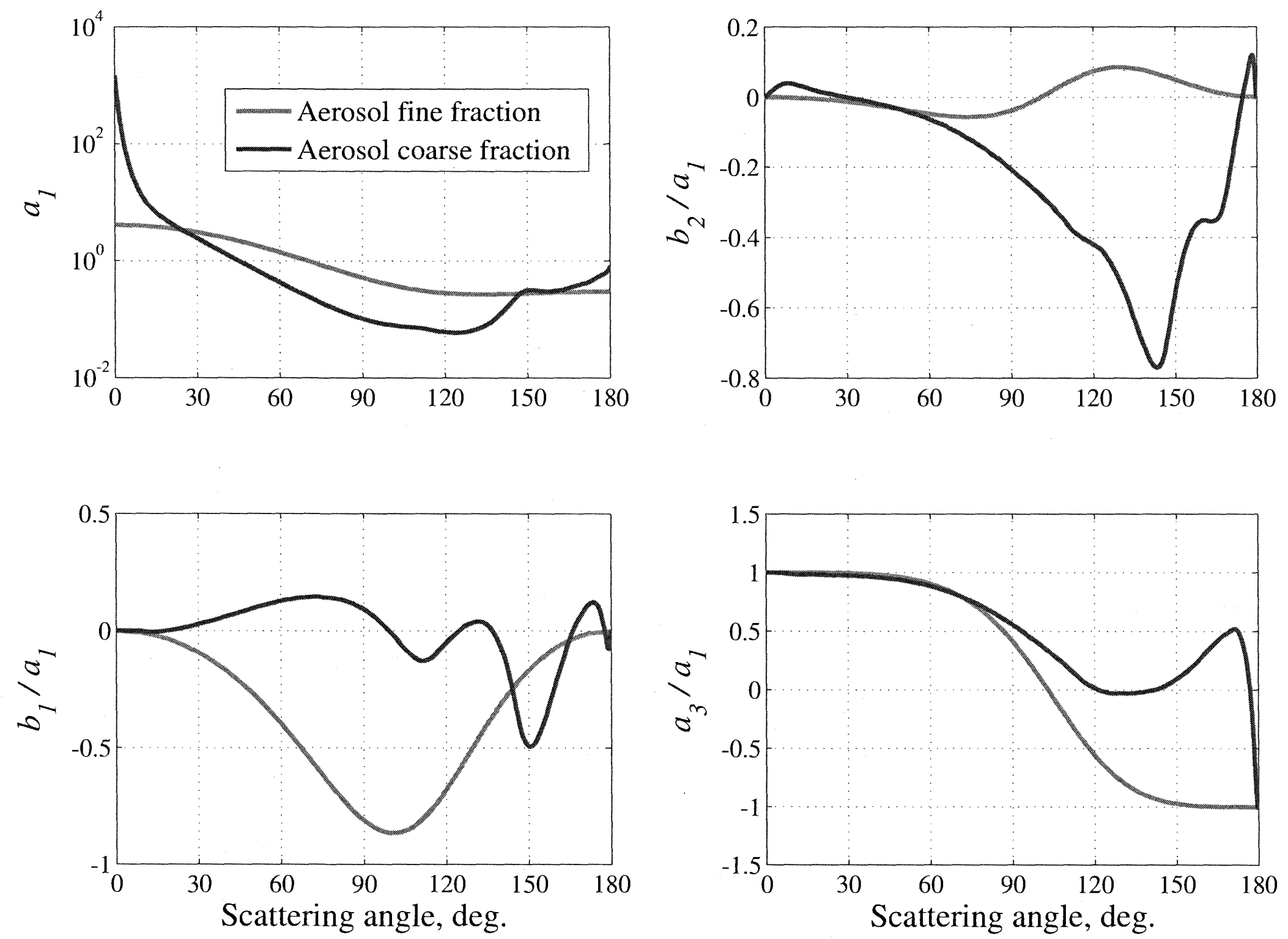

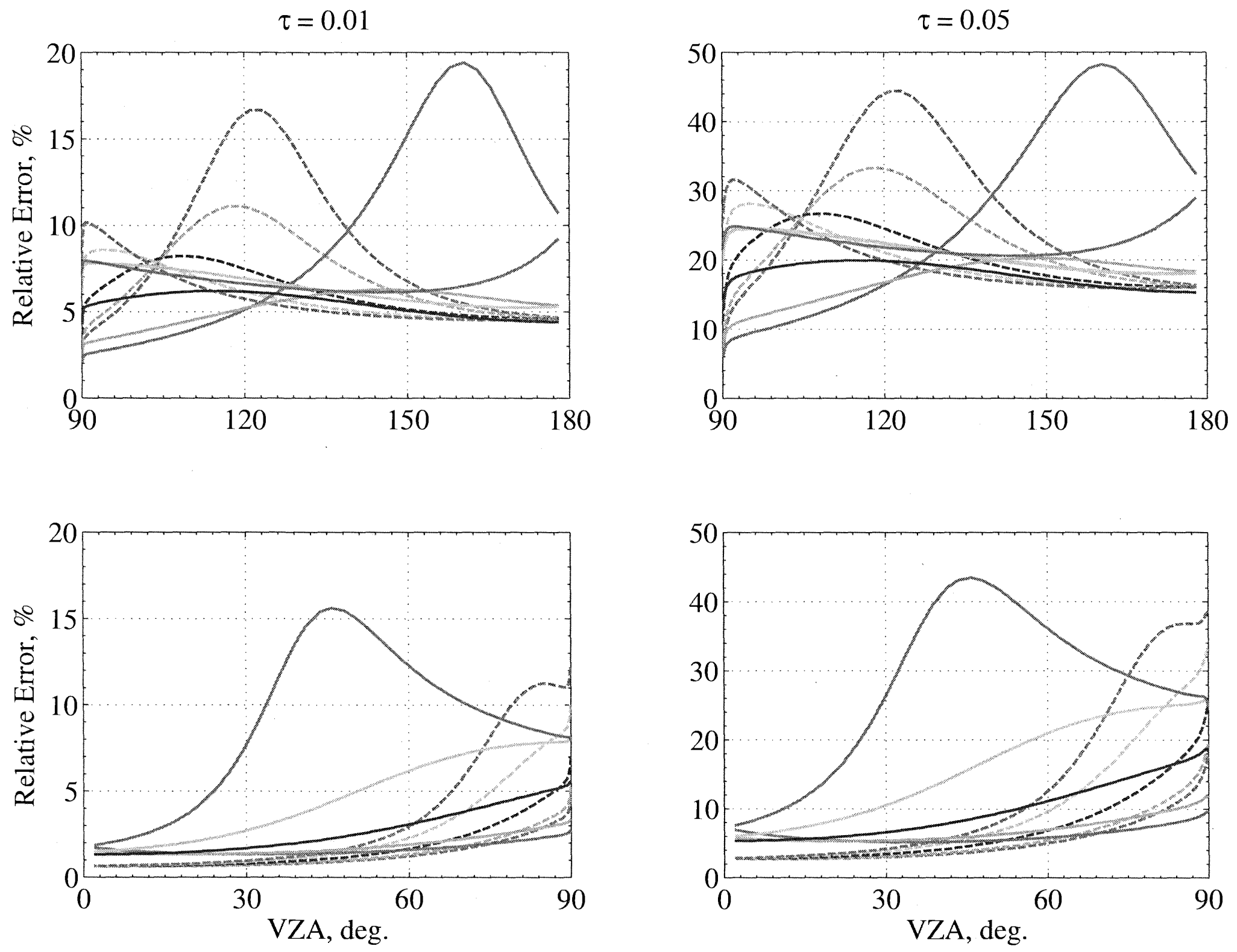

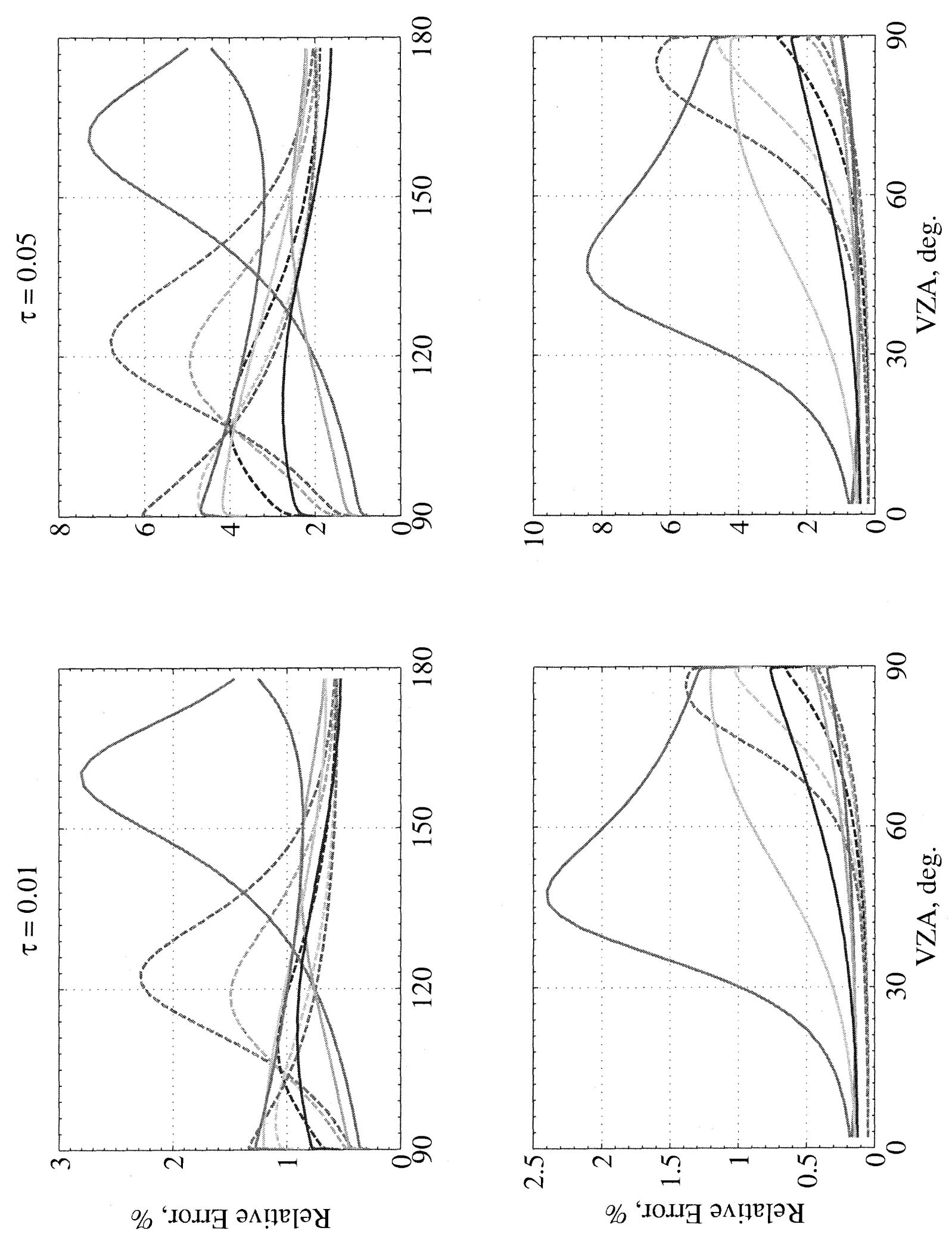

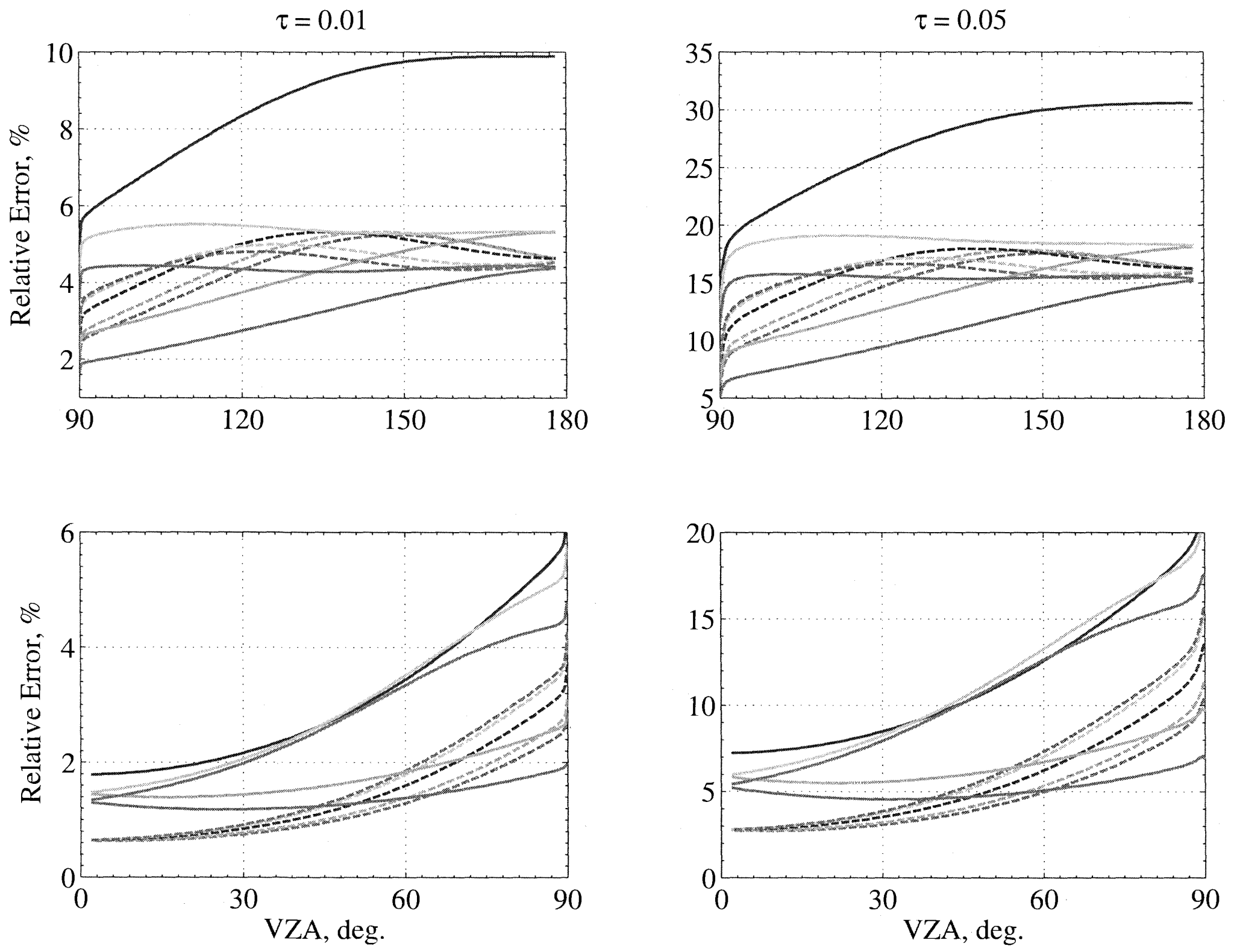

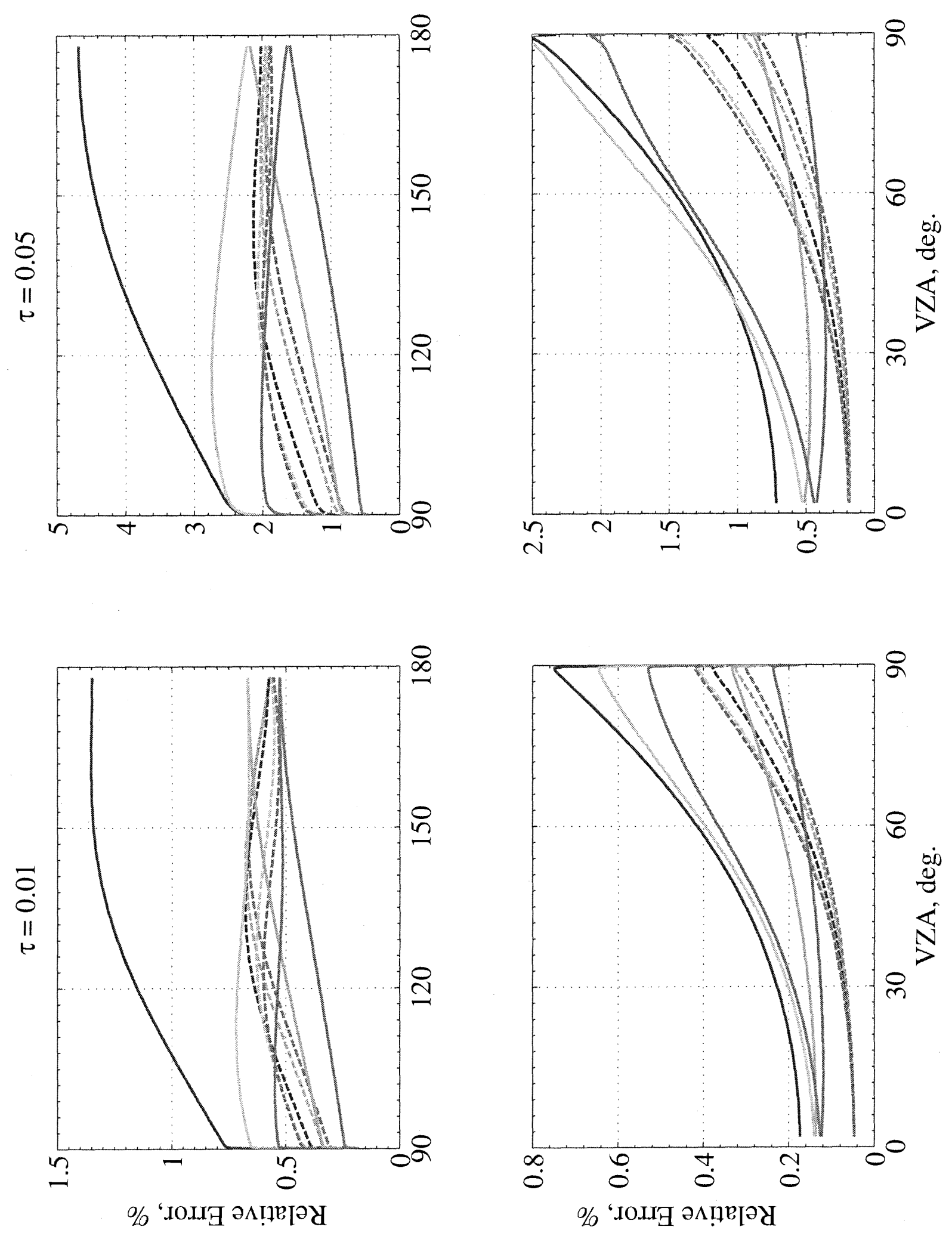


$\begin{aligned}-\mathrm{Az}=0^{\circ}, & \mathrm{SZA}=20^{\circ} \\ \mathrm{Az}=45^{\circ}, & \mathrm{SZA}=20^{\circ} \\ \mathrm{Az}=90^{\circ}, & \mathrm{SZA}=20^{\circ} \\ \mathrm{Az}=135^{\circ}, & \mathrm{SZA}=20^{\circ} \\ \mathrm{Az}=180^{\circ}, & \mathrm{SZA}=20^{\circ} \\ \mathrm{Az}=0^{\circ}, & \mathrm{SZA}=60^{\circ} \\ \mathrm{Az}=45^{\circ}, & \mathrm{SZA}=60^{\circ} \\ \mathrm{Az}=90^{\circ}, & \mathrm{SZA}=60^{\circ} \\ \mathrm{Az}=135^{\circ}, & \mathrm{SZA}=60^{\circ} \\ \mathrm{Az}=180^{\circ}, & \mathrm{SZA}=60^{\circ}\end{aligned}$



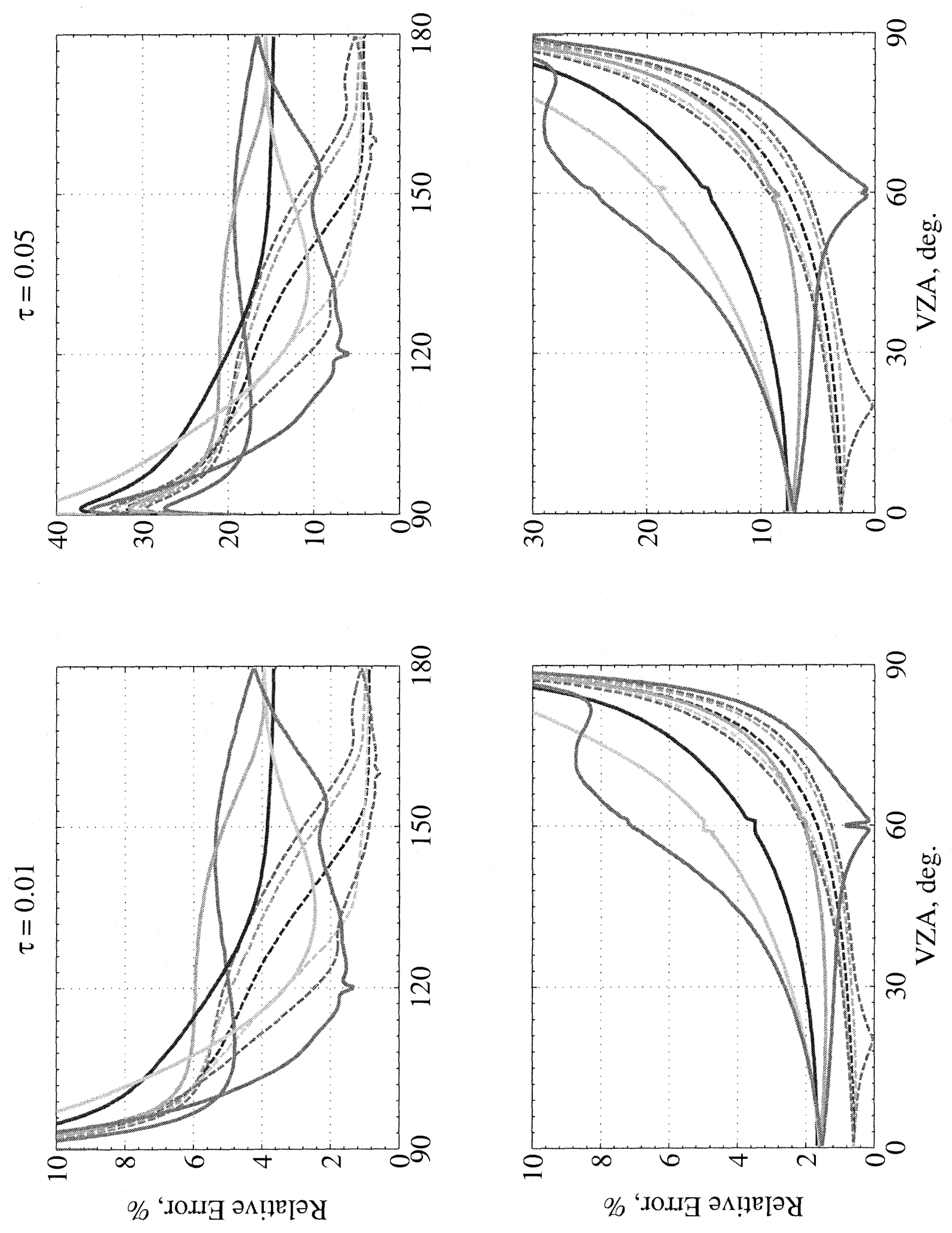

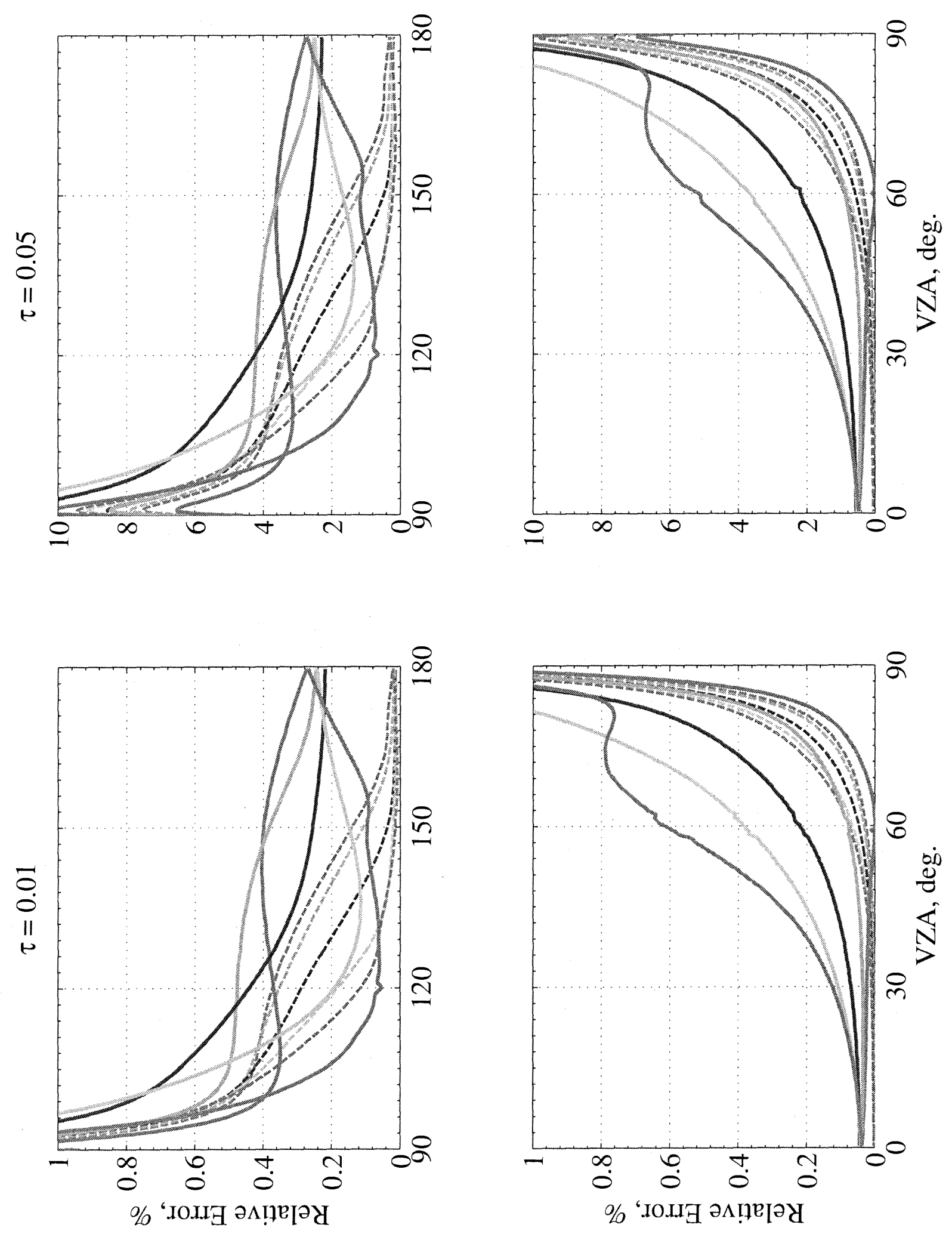

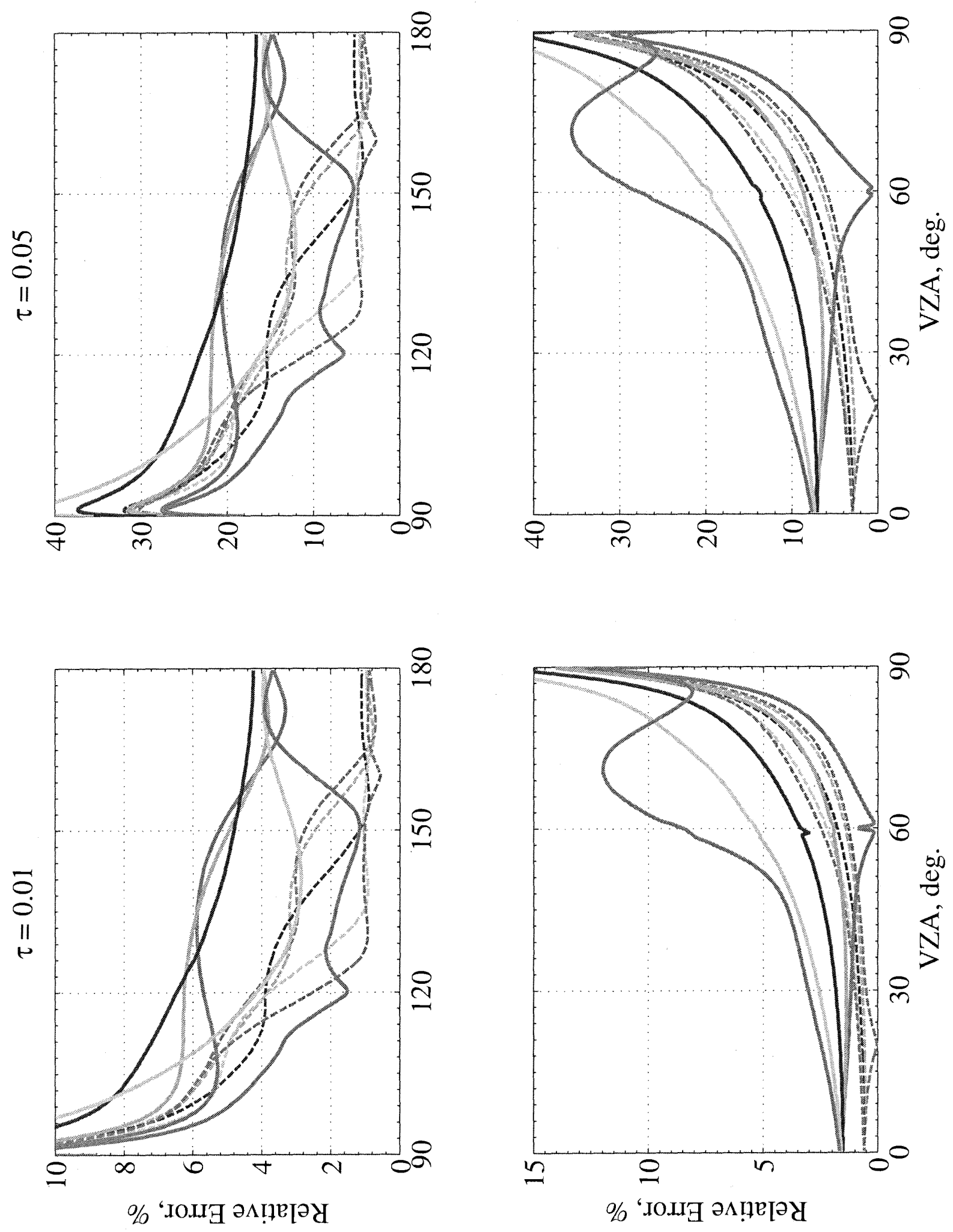

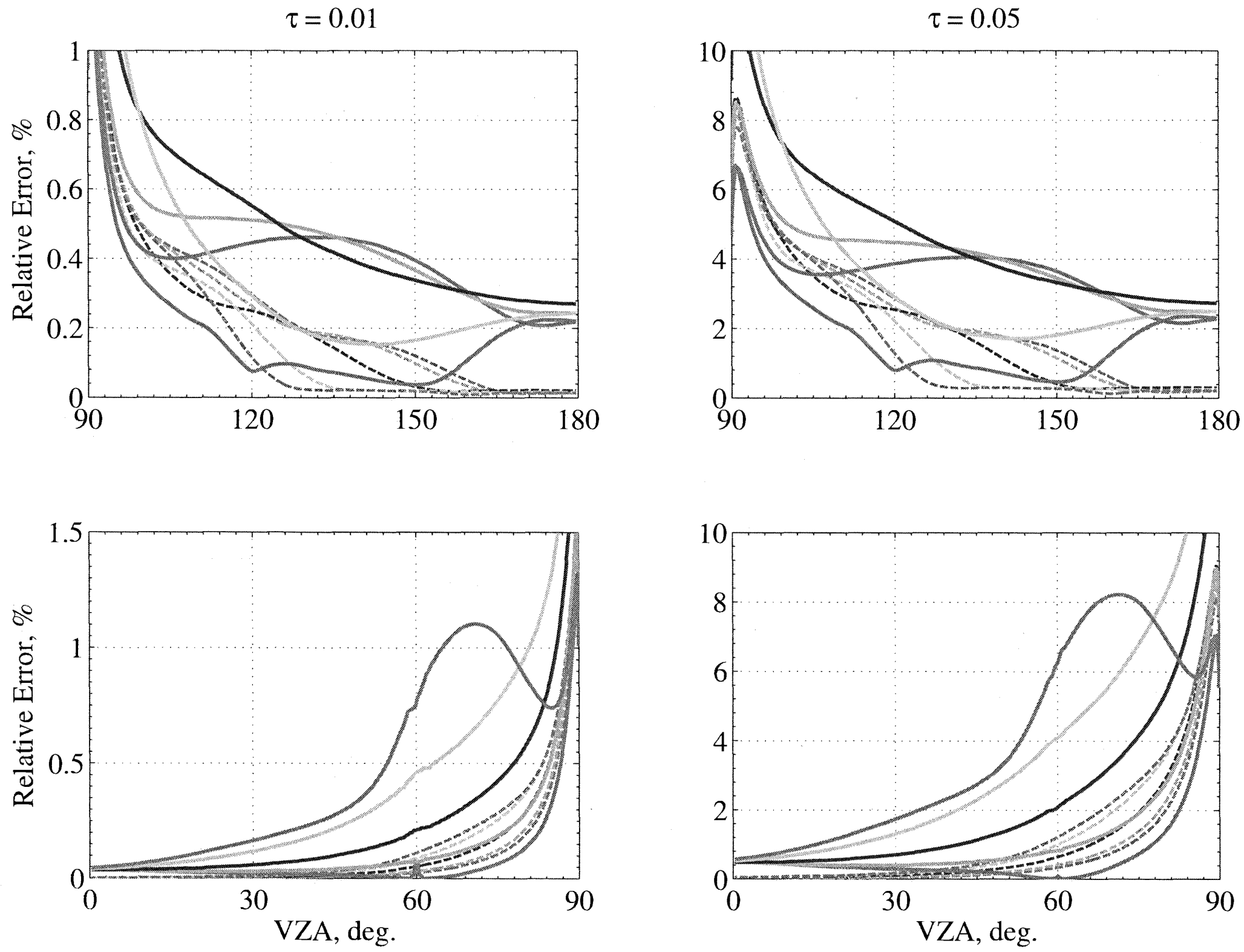

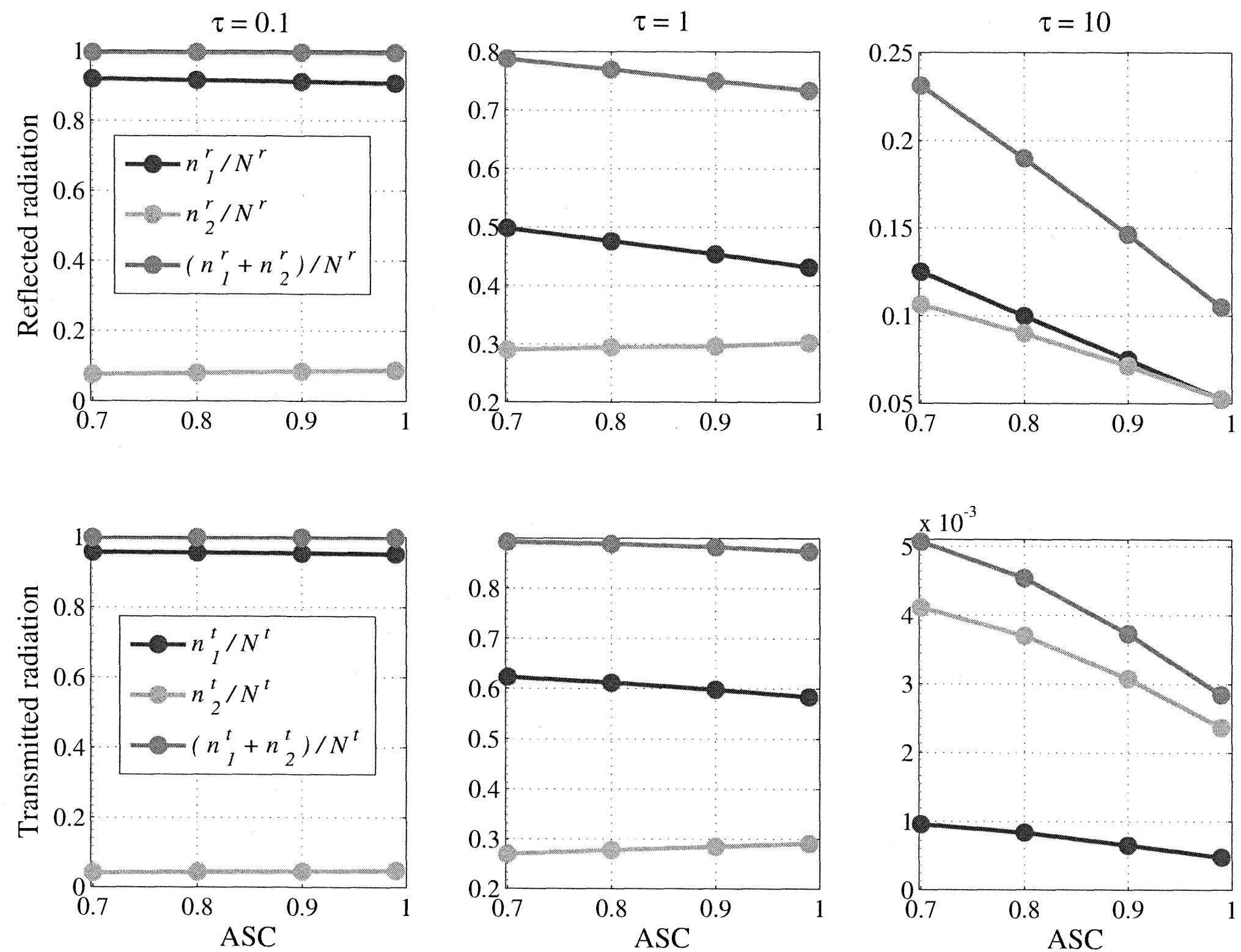

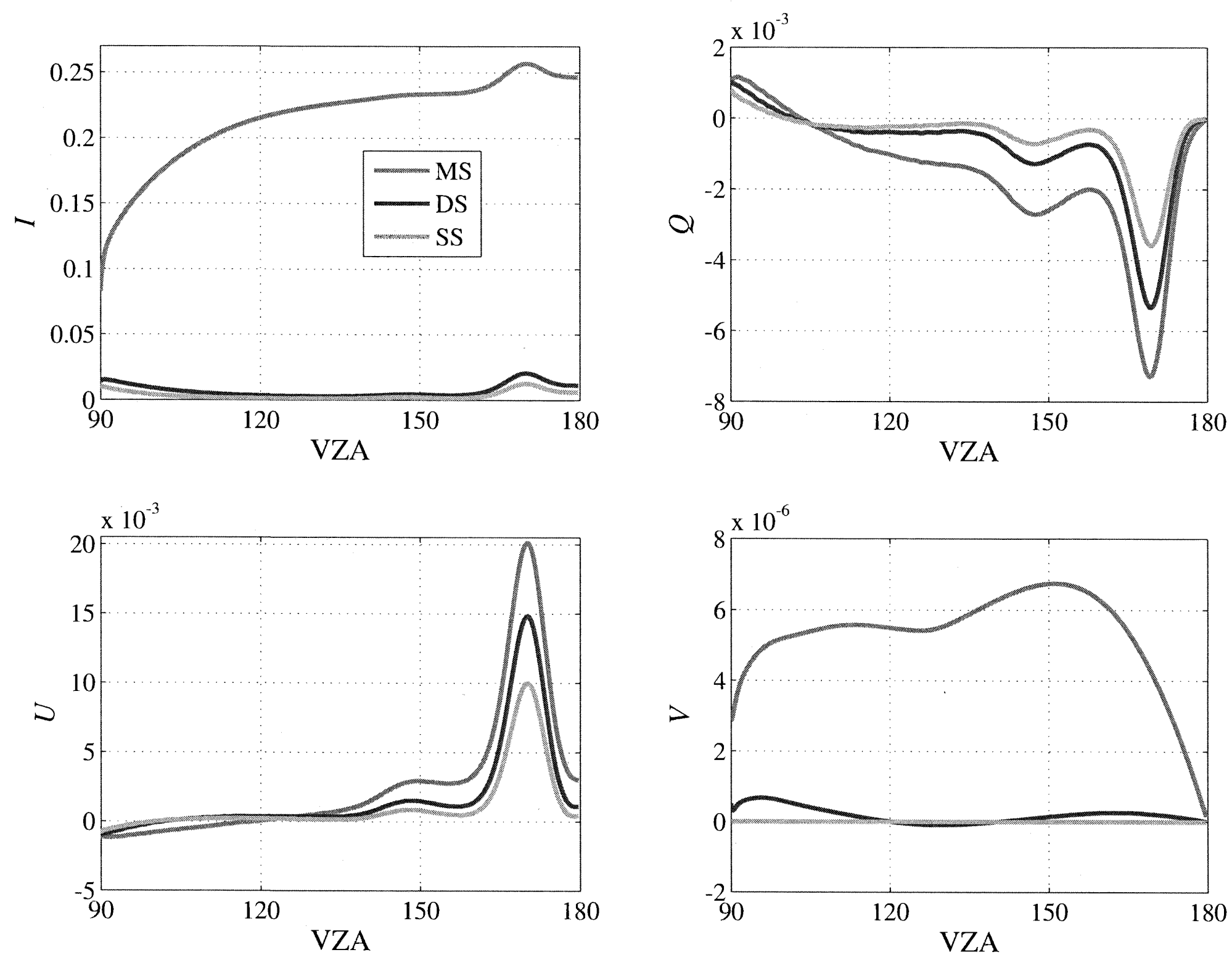

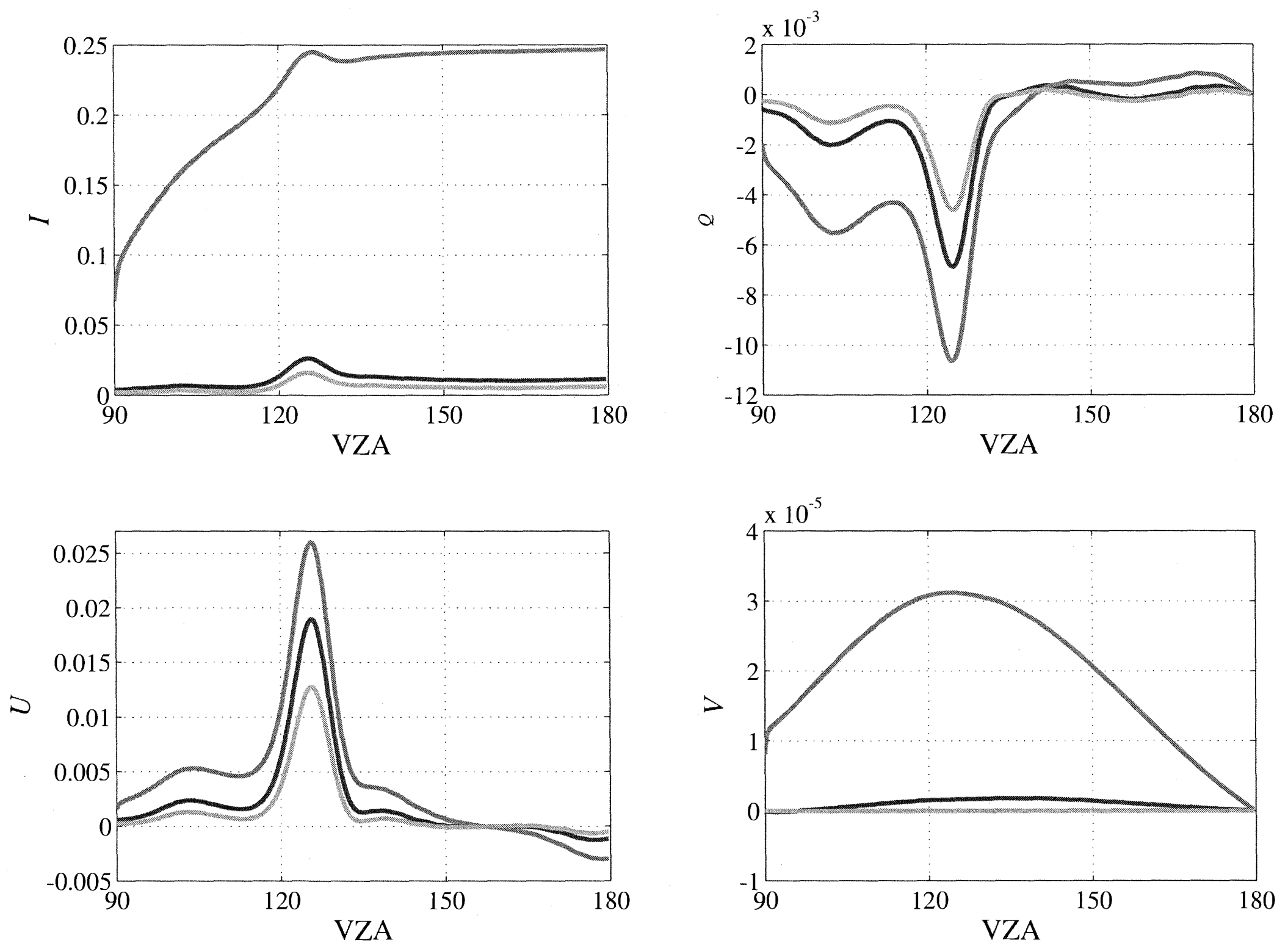
1. The double scattering model has accuracy below $0.2-0.5 \%$ at $\tau<0.01$ in the conservative case;

2. The error rapidly grows with optical depth, so the full VRTE solution is required for the practical aerosol remote sensing;

3. At low optical depths, the relative error for low scattering orders weakly depends on the scattering anisotropy. 